Med Klin Intensivmed Notfmed 2021 · 116:102-103 https://doi.org/10.1007/s00063-021-00787-3 Angenommen: 19. Januar 2021

(c) Springer Medizin Verlag GmbH, ein Teil von Springer Nature 2021

\section{S. John ${ }^{1} \cdot$ M. Kochanek ${ }^{2}$}

${ }^{1}$ Abteilung Internistische Intensivmedizin, Medizinische Klinik 8, Paracelsus Medizinische Privatuniversität Nürnberg und Universität Erlangen-Nürnberg, Klinikum Nürnberg-Süd, Nürnberg, Deutschland

${ }^{2}$ Klinik I für Innere Medizin, Universitätsklinikum Köln, Köln, Deutschland

\title{
Der immunsupprimierte Patient auf der Intensivstation
}

Die Aufnahme von immunsupprimierten Patienten auf die Intensivstation (ICU) stellt meistens jede intensivmedizinische Station vor besondere Herausforderungen. Das liegt zum einen an der Komplexität der Grunderkrankung mit immer komplexer werdenden Therapieoptionen, zum anderen an den vielen begleitenden Komorbiditäten und nicht zuletzt an dem zur Intensivaufnahme führenden akuten Aufnahmegrund dieser Patienten. Häufig sind es besonders schwere Infektionen, oft verbunden mit gleichzeitiger Isolationspflicht der Patienten.

Eine Stichtagsprävalenzstudie zeigte, dass fast jeder 4. Patient auf deutschen Intensivstationen eine Krebserkrankung hat und damit auch in vielen Fällen immunsupprimiert ist [1]. Die intensivmedizinische Betreuung von Patienten mit malignen Erkrankungen hat in den letzten 20 Jahren dabei einen Paradigmenwechsel erfahren. Frühere Ablehnung, dann Zurückhaltung ist über ein vorsichtiges „Herantasten“ mittlerweile in Routine übergegangen, diese Patientengruppe intensivmedizinisch $\mathrm{zu}$ versorgen [2]. Abgesehen von spezifischen, mit der malignen Grundkrankheit assoziierten Notfällen oder postoperativer Überwachung sind die Gründe für die Aufnahme von Patienten mit hämatologischer oder onkologischer Grunderkrankung akute, meistens lebensbedrohliche respiratorische und infektiologische Probleme, seltener auch Blutungen oder neurologische Krisen [1]. Durch die Möglichkeiten der modernen intensivmedizinischen Versorgung und therapeutischen Interventionen und aufgrund der rasanten Entwicklung der antineoplastischen
Therapieoptionen hat sich die Prognose der Krebserkrankung deutlich verbessert [3]. Die kurzfristige ICU-Prognose wird dabei nicht von der Krebserkrankung bestimmt, sondern hängt von der intensivmedizinischen Diagnose ab, wohingegen die langfristige Prognose von der Krebserkrankung abhängt.

Alle diese Informationen stellen den Intensivmediziner vor eine Reihe von spezifischen Fragen im Zusammenhang mit dieser speziellen Patientengruppe. Was beutet das für den Intensivmediziner, was sollte er wissen und wie sieht die Behandlung am Bett aus?

Neben den medizinischen Fragen sollen in diesem Themenheft aber auch Fragen der Therapieziele und -möglichkeiten und die damit verbundenen Fragen zur Aufnahmeindikation auf die Intensivstation diskutiert werden. Gerade auch bei knappen Intensivkapazitäten, wie z. B. jetzt in der SARS-CoV-2-Pandemie, ist diese Frage brisanter denn je.

In Steigerung der Komplexität stellen die Kollegen/innen Beutel und Lueck aus Hannover zuerst den "gewöhnlichen“ Krebspatienten auf der Intensivstation vor. Knochenmarktransplantierte Patienten werden von den Kollegen/innen Stecher et al. aus München dargestellt. Eine ganz besonders komplexe Gruppe stellen Patienten, die mit „chimeric antigen receptor"(CAR)-T-Zellen behandelt werden, dar, da das klinische Bild sehr bunt ist und viele Syndrome streifen kann. Die Kollegen/innen Garcia-Borrega et al. aus Köln berichten über diese neue Behandlungsoption und die dabei ablaufenden pathophysiologischen Veränderungen und Therapieoptionen. Zurzeit findet die Behandlung dieser 
CAR-T-Zell-Patienten in speziellen Therapiezentren statt, aber es ist damit $\mathrm{zu}$ rechnen, dass sich das in Zukunft auch flächendeckend ausbreiten wird.

Last, but not least wird uns ein Krankheitsbild von den Kollegen Eichenauer et al. aus Köln/Berlin/Stuttgart vorgestellt, was sicher jeder schon häufig gesehen, es aber als solches nicht immer wahrgenommen hat: die hämophagozytische Lymphohistiozytose (HLH). Eine wahrscheinlich vollkommen unterschätzte Erkrankung oder besser gesagt ein Syndrom, das wie ein Chamäleon auf der Intensivstation, sicher nicht häufig, aber dafür oft mit einem tödlichen Ausgang, auftritt. Der Beitrag soll dem Intensivmediziner helfen, die Diagnose einer HLH sicher $\mathrm{zu}$ stellen und das Therapiemanagement rasch einleiten $\mathrm{zu}$ können.

Mittlerweile haben sich Strukturen für alle diese sehr speziellen Gruppen hämato-/onkologischer Patienten auf der Intensivstation etabliert. Die iCHOP-Arbeitsgruppe innerhalb der DGIIN freut sich über reges Interesse, Mitarbeit oder Teilnahme an Studien. Interessierte sind herzlich zur Kontaktaufnahme über die DGIIN eingeladen.

Das vorliegende Themenheft will versuchen, für diese speziellen, immunsupprimierten Patienten (Krebspatienten, HLH) Informationen, Hilfen, Antworten und Diskussionsgrundlagen $\mathrm{zu}$ geben. Wir freuen uns, wenn wir Ihnen einen interessanten und informativen Überblick zusammengestellt haben.

Ihr

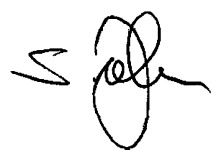

Stefan John

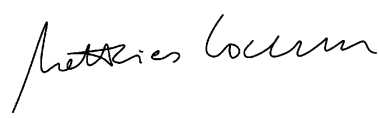

Matthias Kochanek

\section{Korrespondenzadresse}

\section{Prof. Dr. S. John}

Abteilung Internistische Intensivmedizin, Medizinische Klinik 8, Paracelsus Medizinische Privatuniversität Nürnberg und Universität Erlangen-Nürnberg, Klinikum Nürnberg-Süd Breslauer Str. 20, 90471 Nürnberg, Deutschland stefan.john@klinikum-nuernberg.de

\section{PD Dr. M. Kochanek}

Klinik I für Innere Medizin, Universitätsklinikum Köln

Kerpener Str. 62, 50937 Köln, Deutschland matthias.kochanek@uk-koeln.de

Interessenkonflikt. S. John und M. Kochanek geben an, dass kein Interessenkonflikt besteht.

\section{Literatur}

1. Kochanek M, Shimabukuro-Vornhagen A, Russ K et al (2020) Prevalence of cancer patients in German intensive care units. Med Klin Intensivmed Notfmed 115:312-319. https://doi.org/10.1007/ s00063-019-0594-3

2. Azoulay E, Soares M, Darmon M et al (2011) Intensive care of the cancer patient: recent achievements and remaining challenges. Ann Intensive Care 1:5. https://doi.org/10.1186/21105820-1-5

3. Schellongowski P, Beutel G, Kochanek $M$ et al (2016) Intensivmedizinische Aspekte bei hämatologischen und onkologischen Erkrankungen. Intensivmedup2date 12:267-285. https://doi.org/ $10.1055 / \mathrm{s}-0042-103705$

\section{Telemedizin}

Marx, Gernot, Rossaint, Rolf, Marx, Nikolaus (Hrsg.)

2021, XVI, 502 S. 98 Abb., 79,99 EUR ISBN 978-3-662-60610-0

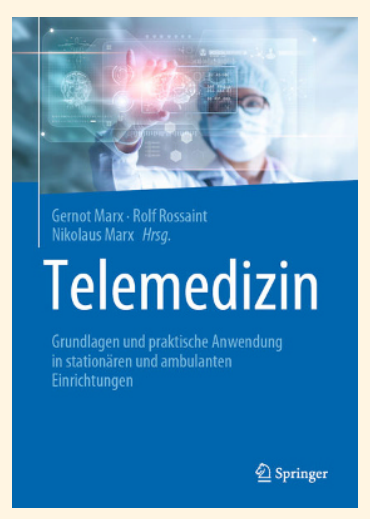

Grundlagen und praktische Anwendung in stationären und ambulanten Einrichtungen:

- Chancen, Risiken und rechtliche Besonderheiten der Telemedizin

- Mit zahlreichen

Anwendungsbeispielen

- Mit Blick in die EU und Nachbarländer

Das Buch gibt einen umfassenden Überblick über den aktuellen Stand und die Entwicklungen im Bereich der Telemedizin in Deutschland. Das Herausgeberteam aus einem der führenden Telemedizinzentren Deutschlands und ein interdisziplinäres Autorenteam liefern wesentliche Grundlagen sowie zahlreiche Anwendungsbeispiele aus dem stationären und ambulanten Bereich. Zudem werden Chancen und Risiken der Telemedizin erörtert, so dass der Leser gut für die Zukunft in Klinik oder Praxis gerüstet ist. Ein Grundlagen- und Nachschlagewerk für alle Ärzte, die sich für digitale Zukunft im Gesundheitswesen wappnen und ihre Patienten optimal versorgen möchten. 